Sādhanā Vol. 39, Part 1, February 2014, pp. 139-147. (C) Indian Academy of Sciences

\title{
A reexamination of some puzzling results in linearized elasticity
}

\author{
$\mathrm{C} \mathrm{S} \mathrm{JOG}^{1, *}$ and HARISH P CHERUKURI ${ }^{2}$ \\ ${ }^{1}$ Department of Mechanical Engineering, Indian Institute of Science, \\ Bangalore 560 012, India \\ ${ }^{2}$ Department of Mechanical Engineering and Engineering Science, \\ University of North Carolina at Charlotte, Charlotte, NC 28223-0001, USA \\ e-mail: jogc@mecheng.iisc.ernet.in; hcheruku@uncc.edu
}

MS received 11 September 2012; revised 24 April 2013; accepted 14 May 2013

\begin{abstract}
In this paper, we analyse three commonly discussed 'flaws' of linearized elasticity theory and attempt to resolve them. The first 'flaw' concerns cylindrically orthotropic material models. Since the work of Lekhnitskii (1968), there has been a growing body of work that continues to this day, that shows that infinite stresses arise with the use of a cylindrically orthotropic material model even in the case of linearized elasticity. Besides infinite stresses, interpenetration of matter is also shown to occur. These infinite stresses and interpenetration occur when the ratio of the circumferential Young modulus to the radial Young modulus is less than one. If the ratio is greater than one, then the stresses at the center of a spinning disk are found to be zero (recall that for an isotropic material model, the stresses are maximum at the center). Thus, the stresses go abruptly from a maximum value to a value of zero as the ratio is increased to a value even slightly above one! One of the explanations provided for this extremely anomalous behaviour is the failure of linearized elasticity to satisfy material frameindifference. However, if this is the true cause, then the anomalous behaviour should also occur with the use of an isotropic material model, where, no such anomalies are observed. We show that the real cause of the problem is elsewhere and also show how these anomalies can be resolved. We also discuss how the formulation of linearized elastodynamics in the case of small deformations superposed on a rigid motion can be given in a succinct manner. Finally, we show how the long-standing problem of devising three compatibility relations instead of six can be resolved.
\end{abstract}

Keywords. Linearized elasticity; singularities; compatibility; orthotropy; isotropy; elastodynamics.

*For correspondence 


\section{Introduction}

Lekhnitskii (1968) appears to be the first to observe that stress singularities can arise at the center of a cylindrically orthotropic cylinder subjected to pressure loading on the outer boundary. Since then, there has been a growing literature on this 'anomalous' behaviour, which we now briefly summarize (note that these anomalies do not arise when the material is isotropic). Let $E_{r}$ and $E_{\theta}$ denote the Young moduli in the radial and circumferential directions, respectively, and let $n$ denote the ratio $E_{\theta} / E_{r}$. Tang (1969) and Jain et al (2000) showed that infinite radial stresses occur at the center of a solid flat rotating disk when $n<1$, while the radial and hoop stresses are both zero at the center when $n>1$. It is well-known that these stresses are maximum at the center when $n=1$. Thus, there is an abrupt transition from a maximum state of stress to zero as $n$ increases from 1 to $1+\epsilon$, where $\epsilon$ is an arbitrarily small positive number! Galmudi \& Dvorkin (1995), and Horgan \& Baxter (1996) showed that when a hollow cylinder (or disk) is subjected to a uniform distributed pressure on its outer boundary with its inner boundary traction-free, the stress concentration factor was infinity when $n<1$. Similar anomalous results were also shown in the case of pressurized hollow spheres in Ting (1999a), and cylindrical tubes under loading conditions different from pressure loading in Ting (1999b). Fosdick \& RoyerCarfagni (2001), Fosdick et al (2008) noted that in the compressed disk problem of Lekhnitskii (1968), in addition to infinite stresses, there are also areas where interpenetration of matter occurs when $n<1$. The authors impose a constraint of local injectivity to prevent this interpenetration. Freddi \& Royer-Carfagni (2009) also reported infinite stresses and interpenetration in an aeolotropic disk whose boundary is subjected to Dirichlet boundary conditions equivalent to a rigid body displacement. They attributed the reason to the failure of linearized elasticity to satisfy material frame-indifference (Podio-Guidugli 1987). However, if the failure to satisfy material frame-indifference is really the root cause, then it is not clear why such anomalous behaviour is not seen in the case of isotropic materials, where also material frame-indifference is not satisfied. Among all the works in the literature, the work by Tarn (2002) is perhaps the only one that first states that 'unbounded stress is physically unacceptable', and then correctly identifies the source of the problem as the conflicting definition of anisotropy at $r=0$. Unfortunately, the solution that is suggested to address this problem is to use an outer cylinder that is cylindrically anisotropic and an inner core that is transversely isotropic. This does not resolve the problem, since in the limit as the inner core is shrunk to zero, the problem reappears (Fosdick et al 2008). We provide a resolution of this problem in section 2 .

Tortorelli (2011) has recently presented a generalization of the conventional theory of linearized elastodynamics that addresses the case of small displacement gradients superposed on a rigid motion. We show in section 3 that such a formulation can be presented very succinctly by simply considering a frame of reference attached to the rigid motion.

In section 4, we present a resolution of the problem of formulating three (nonlinear) compatibility relations instead of the six (linear) relations that are usually presented. Conclusions are provided in section 5 .

\section{Resolution of the stress singularity problem}

Although it is true that linearized elasticity does not satisfy material frame-indifference, this is not so serious a flaw to cause stress singularities or interpenetration of matter (even in problems with smooth loading and no cracks) as claimed. We show in this work that the real cause of such behaviour is the use of defective constitutive relations and not an intrinsic flaw with linearized elasticity. In fact, since $|\nabla \boldsymbol{u}|$ is assumed to be small, it is inconceivable that $\operatorname{det} \boldsymbol{F}=\operatorname{det}(\boldsymbol{I}+\boldsymbol{\nabla} \boldsymbol{u})$ 
(where $\boldsymbol{u}$ and $\boldsymbol{F}$ denote the displacement field and deformation gradient, respectively) can become negative, which is the condition for interpenetration to occur. Since these stress singularities arise mostly with the use of a cylindrically aeolotropic or cylindrically orthotropic material model, we first describe this model. For planar problems, the constitutive relations are given by

$$
\begin{aligned}
\tau_{r r} & =c_{11}(r) \epsilon_{r r}+c_{12}(r) \epsilon_{\theta \theta}, \\
\tau_{\theta \theta} & =c_{21}(r) \epsilon_{r r}+c_{22}(r) \epsilon_{\theta \theta}, \\
\tau_{r \theta} & =2 c_{66}(r) \epsilon_{r \theta},
\end{aligned}
$$

where $\boldsymbol{\tau}$ and $\boldsymbol{\epsilon}$ denote the stress and strain fields, and $c_{11}(r), c_{22}(r), c_{12}(r)=c_{21}(r)$ and $c_{66}(r)$ are the elastic parameters that depend only on the radial direction $r$, but not on the angular direction $\theta$. The inverse relations are

$$
\begin{aligned}
\epsilon_{r r} & =\frac{1}{E_{r}(r)} \tau_{r r}-\frac{\nu_{r}(r)}{E_{r}(r)} \tau_{\theta \theta}, \\
\epsilon_{\theta \theta} & =-\frac{\nu_{\theta}(r)}{E_{\theta}(r)} \tau_{r r}+\frac{1}{E_{\theta}(r)} \tau_{\theta \theta}, \\
\epsilon_{r \theta} & =\frac{1}{2 G(r)} \tau_{r \theta},
\end{aligned}
$$

where

$$
\begin{aligned}
c_{11}(r) & =\frac{E_{r}(r)}{1-v_{r}(r) v_{\theta}(r)}, & c_{22}(r) & =\frac{E_{\theta}(r)}{1-v_{r}(r) v_{\theta}(r)}, \\
c_{12}(r) & =\frac{E_{\theta}(r) v_{r}(r)}{1-v_{r}(r) v_{\theta}(r)}, & c_{21}(r) & =\frac{E_{r}(r) v_{\theta}(r)}{1-v_{r}(r) v_{\theta}(r)}, \\
c_{66}(r) & =G(r) . & &
\end{aligned}
$$

The symmetry requirement $c_{12}(r)=c_{21}(r)$ imposes the constraint

$$
\frac{v_{r}(r)}{E_{r}(r)}=\frac{v_{\theta}(r)}{E_{\theta}(r)}
$$

In addition, the positive-definiteness of the elasticity tensor imposes the restrictions

$$
\begin{array}{llll}
c_{11}(r)>0, & c_{22}(r)>0, & c_{66}(r)>0, & c_{11}(r) c_{22}(r)-c_{21}(r) c_{12}(r)>0, \\
E_{r}(r)>0, & E_{\theta}(r)>0, & G(r)>0, & 1-v_{r}(r) v_{\theta}(r)>0 .
\end{array}
$$

Just as in the case of an isotropic material, one deduces the bounds $\mu>0$ and $3 \lambda+2 \mu>0$ for the Lamé constants $\lambda$ and $\mu$ based on certain special types of loadings (see, e.g., pg. 123 of Ciarlet (1988)), we also use certain special loading conditions to deduce further constraints on the material constants in the above constitutive relations. Consider the radially symmetric deformation of a cylindrically orthotropic solid cylinder due to loading by a uniform pressure acting normal to its boundary. As noted by Tarn (2002), at $r=0$, the tangential direction at $\theta$ is the radial direction at $\theta+\pi / 2$, and vice versa, so that $\left.\tau_{\theta \theta}\right|_{\substack{r=0 \\ \theta}}=\left.\tau_{r r}\right|_{\substack{r=0 \\ \theta+\pi / 2}}$. Since the deformation is radially symmetric, $\tau_{r r}$ and $\tau_{\theta \theta}$ are independent of $\theta$, so that we get $\left.\tau_{r r}\right|_{r=0}=$ $\left.\tau_{\theta \theta}\right|_{r=0}$ (in this regard, we mention that Timoshenko and Goodier (1970) while discussing the 
problem of a rotating disk state in a footnote 'It can be seen from the definitions of $\tau_{r r}, \tau_{\theta \theta}$ that when they are independent of $\theta$, they must be equal at the center'). In a similar manner, we also get $\left.\epsilon_{r r}\right|_{r=0}=\left.\epsilon_{\theta \theta}\right|_{r=0}$. From Eq. (1), we see that this imposes the constraint

$$
\left.\left.c_{11}\right|_{r=0}=\left.c_{22}\right|_{r=0} \quad \text { (alternatively, } \frac{c_{11}}{c_{22}}=g(r), \text { where } g(0)=1\right),
$$

which in turn implies that $E_{r}=E_{\theta}$ and $v_{r}=v_{\theta}$ at $r=0$. Thus, at the axis $r=0$, the cylindrically orthotropic material model should reduce to an isotropic model and to a transversely isotropic model in the planar and three-dimensional cases, respectively. Of course, in case the body is assumed to be homogeneous, the respective models are isotropic and transversely isotropic everywhere; thus, it is essential to have inhomogeneity in order to get a cylindrical, or, in general, curvilinear, orthotropic model. In the case of a spherically orthotropic model, using $\left.\epsilon_{r r}\right|_{r=0}=\left.\epsilon_{\theta \theta}\right|_{r=0}=\left.\epsilon_{\phi \phi}\right|_{r=0}$ and $\left.\tau_{r r}\right|_{r=0}=\left.\tau_{\theta \theta}\right|_{r=0}=\left.\tau_{\phi \phi}\right|_{r=0}$, we again get an inhomogeneous material model that reduces to an isotropic model at the center (if the sphere is constrained to be homogeneous, then it has to be isotropic everywhere). Since, similar to the constraints on the Lamé constants, these are also material constraints, they should be imposed even in problems where the axis or center is not a part of the domain, such as, for example, in problems involving hollow cylinders or spheres, or even when the loading is not radially symmetric. The singularities or infinite stress concentration factors in the solutions arise since these constraints are not imposed. In fact, as seen from the solutions presented in figures 2 and 3 in Jain et al (1999), there are no singularities in the stresses for the particular choice of material constants that the authors have used, which obey the constraint of Eq. (3). Another benefit of imposing the constraint $v_{r}=v_{\theta}$ at $r=0$, is that it imposes bounds on the individual Poisson ratios $v_{r}$ and $v_{\theta}$, which, individually, can become unbounded if the only constraint on them is that imposed by the condition $1-v_{r}(r) v_{\theta}(r)>0$ in Eq. (2b).

Based on the above discussion, we make the following remarks regarding the observations in Freddi \& Royer-Carfagni (2009):

(i) In the composite isotropic disk considered in section (3.3) of Freddi \& Royer-Carfagni (2009), the linearized stresses do tend to zero as the angle of rigid rotation tends to zero, thus, approaching the correct nonlinear stress limit.

(ii) For the aeolotropic disk problem considered in section (3.4) of Freddi \& Royer-Carfagni (2009) (with similar constitutive relations being used in Ting (1999a, b), Fosdick \& RoyerCarfagni (2001), Fosdick et al (2008)), as mentioned above, singularities arise as a result of the constraint given by Eq. (3) not being imposed.

(iii) For the functionally graded material considered in section (3.5) of Freddi \& Royer-Carfagni (2009), the Young modulus tends to zero as $r$ tends to zero, leading to a loss of ellipticity at the origin, as noted by the authors themselves.

In conclusion, the highly anomalous behaviour of solutions observed with anisotropic linear elastic materials (stress singularities, cavitation, interpenetration, etc.) seems to be due to flaws in the particular constitutive models being used, rather than due to any intrinsic shortcoming of linearized elasticity.

\section{Generalized formulation of elastodynamics: Small on rigid}

Tortorelli (2011) developed a formulation that is a generalization of the conventional theory of linearized elastodynamics; one of the goals of developing this formulation as stated by the author 
is to eliminate the anomalous behaviour of anisotropic bodies that was dealt with in the previous section. Tortorelli (2011) first states that the conventional theory 'addresses the case of motions that have small displacement gradients with respect to a reference configuration of the elastic body that is unstressed and at rest'. He then proposed a generalization (via a very complicated derivation) that addresses the case where the linearization is with respect to a reference configuration that is in rigid motion, i.e., the generalization addresses the case where the displacement gradients with respect to the underlying rigid motion are small. We first show that, counter to the claim in Tortorelli (2011), the conventional theory is not restricted to the case of a reference configuration that is at rest. Consider a frame of reference $O$ that is in arbitrary motion with respect to another frame of reference $O^{*}$ as shown in figure 1. Let $c(t)$ be the vector connecting the origins of these two coordinate systems as shown in figure 1 . Let $\boldsymbol{e}_{i}^{*}$ and $\boldsymbol{e}_{i}, i=1,2,3$, be the basis vectors associated with the $O^{*}$ and $O$ frames of reference, and let $\boldsymbol{Q}=\boldsymbol{e}_{k} \otimes \boldsymbol{e}_{k}^{*}$. If $\boldsymbol{x}^{*}$ and $\boldsymbol{x}$ are the position vectors of a point with respect to the $O^{*}$ and $O$ frames of reference, then we have Marsden and Hughes (1983)

$$
\boldsymbol{x}^{*}=\boldsymbol{Q}(t) \boldsymbol{x}+\boldsymbol{c}(t)
$$

By imposing the principle of frame-indifference, whereby the 'governing equations have the same form in all frames of reference' Marsden and Hughes (1983), the body force in the $O$ frame of reference is given by Jog (2007)

$$
\boldsymbol{b}=\boldsymbol{Q}^{T}\left[\boldsymbol{b}^{*}-\ddot{\boldsymbol{c}}\right]-\dot{\boldsymbol{\Omega}} \times \boldsymbol{x}-\boldsymbol{\Omega} \times(\boldsymbol{\Omega} \times \boldsymbol{x})-2 \boldsymbol{\Omega} \times \boldsymbol{v},
$$

where $v$ is the velocity (with respect to the $O$ frame of reference) of the particle whose position vector is $\boldsymbol{x}$, and

$$
\boldsymbol{\Omega}=\left[\begin{array}{c}
\dot{\boldsymbol{e}}_{2} \cdot \boldsymbol{e}_{3} \\
\dot{\boldsymbol{e}}_{3} \cdot \boldsymbol{e}_{1} \\
\dot{\boldsymbol{e}}_{1} \cdot \boldsymbol{e}_{2}
\end{array}\right]
$$

By letting the $O$ frame of reference coincide with the 'rigid' motion, we get the governing equation of elastodynamics as

$$
\rho_{0} \frac{\partial^{2} \boldsymbol{u}}{\partial t^{2}}=\nabla_{X} \cdot \boldsymbol{T}+\rho_{0} \boldsymbol{b}(\boldsymbol{x}, t),
$$

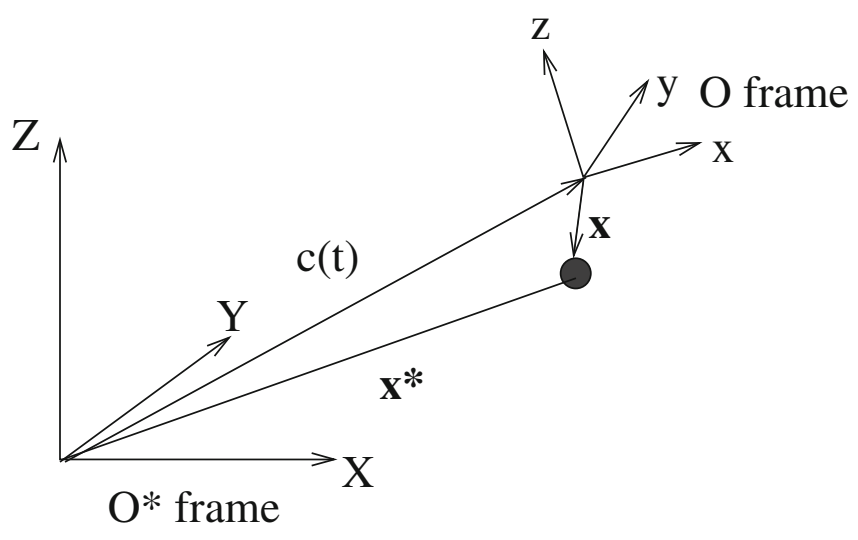

Figure 1. Frames of reference. 
where $\rho_{0}$ is the density in the reference configuration, $\boldsymbol{T}$ denotes the first Piola-Kirchhoff stress with respect to the $O$ frame of reference, and $\boldsymbol{b}$ is given by Eq. (4). To obtain the linearized equations, one linearizes the constitutive relation $\boldsymbol{T}=\hat{\boldsymbol{T}}(\boldsymbol{F})$ to get

$$
\hat{\boldsymbol{T}}(\boldsymbol{F})=\mathbf{C}[\boldsymbol{\epsilon}]+\mathrm{o}(\boldsymbol{\nabla u}),
$$

where $\boldsymbol{\epsilon}=\left[\nabla \boldsymbol{u}+(\nabla \boldsymbol{u})^{T}\right] / 2$, and $\mathbf{C}=D \hat{\boldsymbol{T}}(\boldsymbol{I})$ is the elasticity tensor, and one also linearizes the body force vector to get

$$
\boldsymbol{b}=Q^{T}\left[b^{*}-\ddot{\boldsymbol{c}}\right]-\dot{\boldsymbol{\Omega}} \times \boldsymbol{X}-\boldsymbol{\Omega} \times(\boldsymbol{\Omega} \times \boldsymbol{X})-2 \boldsymbol{\Omega} \times \boldsymbol{v},
$$

where $\boldsymbol{X}$ is the position vector of the point in the reference configuration. Thus, we see that the linearized equations of elastodynamics for the 'small on rigid' case can be derived simply by considering a frame of reference that is attached to the 'rigid' motion.

Another point of departure from the classical treatment in the generalization of Tortorelli (2011) is to not ignore $\boldsymbol{u}$ in comparison to $\boldsymbol{X}$ in the $-\dot{\boldsymbol{\Omega}} \times \boldsymbol{x}-\boldsymbol{\Omega} \times(\boldsymbol{\Omega} \times \boldsymbol{x})$ term in Eq. (4) as is usually done in all the classical treatments, e.g., Timoshenko and Goodier (1970). However, by its very definition, the approximation entailed by the linearization is valid when $\boldsymbol{u}$ is small in comparison with $\boldsymbol{X}$. If $\boldsymbol{u}$ is large then, of course, one cannot neglect $\boldsymbol{u}$ in comparison to $\boldsymbol{X}$, but in such a case, one necessarily needs to use the equations of nonlinear elastodynamics whereby one uses the nonlinear strain-displacement and possibly nonlinear stress-strain relations as well. Thus, not ignoring $\boldsymbol{u}$ in comparison with $\boldsymbol{X}$ within the linearized elastodynamics framework is an unnecessary complication, that in lieu of the added complexity, yields no benefits. In fact, as shown by the author himself, in a disk spinning with angular speed $\omega$, the differences in the results between the 'new' theory and the classical one are indistinguishable for small $\omega$, while for large $\omega$, the results are different, but both, the classical and 'new' theory results are invalid! Linearization is a process whereby the dominant term in each expression is retained, and it is not essential that every term that is linear in $\boldsymbol{u}$ enters into the linearized formulation. In fact, one can say that either linearized theory (classical or new) should not be used precisely when the results of these two theories start differing significantly.

\section{Compatibility relations in linearized elasticity}

We first present some preliminary material. If $S$ is a symmetric tensor, then the spectral decomposition of $\boldsymbol{S}$ can be written in terms of its eigenvalues/eigenvectors $\left(\boldsymbol{\lambda}_{i} \boldsymbol{e}_{i}^{*}\right)$ as Jog (2007)

$$
\boldsymbol{S}=\sum_{i=1}^{3} \lambda_{i} \boldsymbol{e}_{i}^{*} \otimes \boldsymbol{e}_{i}^{*}
$$

The expressions for the principal invariants of $S$ are given by

$$
\begin{aligned}
& I_{1}=\operatorname{tr} S=\lambda_{1}+\lambda_{2}+\lambda_{3} \\
& I_{2}=\frac{1}{2}\left[(\operatorname{tr} S)^{2}-\operatorname{tr}\left(S^{2}\right)\right]=\lambda_{1} \lambda_{2}+\lambda_{2} \lambda_{3}+\lambda_{1} \lambda_{3} \\
& I_{3}=\operatorname{det}(S)=\frac{1}{6}\left[(\operatorname{tr} S)^{3}-3(\operatorname{tr} S)\left(\operatorname{tr} S^{2}\right)+2 \operatorname{tr} S^{3}\right]=\lambda_{1} \lambda_{2} \lambda_{3}
\end{aligned}
$$


If $S$ is zero, then from the characteristic equation

$$
\operatorname{det}(\lambda \boldsymbol{I}-\boldsymbol{S})=\lambda^{3}-I_{1} \lambda^{2}+I_{2} \lambda-I_{3}=0,
$$

it is clear that all the eigenvalues of $S$ are zero. Conversely, if all the eigenvalues $\lambda_{i}$ are zero, then from Eq. (6), it is clear that $\boldsymbol{S}=\mathbf{0}$. Thus, $\boldsymbol{S}$ is zero if and only if all its eigenvalues are zero.

If all the principal invariants of $\boldsymbol{S}$ are zero, then from Eq. (10) it is clear that all the eigenvalues $\lambda_{i}$ are zero. Conversely, if all the eigenvalues are zero, then from Eqs (7)-(9) it follows that all the principal invariants of $S$ are zero. Thus, all the principal invariants of $S$ are zero if and only if all the eigenvalues of $\boldsymbol{S}$ are zero.

If $\operatorname{tr} S=\operatorname{tr} S^{2}=\operatorname{tr} S^{3}=0$, then from Eqs (7)-(9), it immediately follows that $I_{1}=I_{2}=I_{3}=0$. Conversely, if all the principal invariants are zero, then from Eq. (7), we first get $\operatorname{tr} S=0$, followed by $\operatorname{tr}\left(S^{2}\right)=0$ from Eq. (8), followed in turn by $\operatorname{tr}\left(S^{3}\right)=0$ from Eq. (9). Thus, the principal invariants of $S$ are zero if and only if $\operatorname{tr} S=\operatorname{tr} S^{2}=\operatorname{tr} S^{3}=0$.

The curl of a second-order tensor $\boldsymbol{T}$ is defined by

$$
(\nabla \times \boldsymbol{T}) \boldsymbol{u}:=\nabla \times\left(\boldsymbol{T}^{T} \boldsymbol{u}\right) \quad \forall \text { constant vectors } \boldsymbol{u} .
$$

In component form,

$$
(\nabla \times \boldsymbol{T})_{i j}=\epsilon_{i r s} \frac{\partial T_{j s}}{\partial x_{r}} .
$$

From the above relation, we get

$$
[\nabla \times(\nabla \times \boldsymbol{T})]_{i j}=\epsilon_{i m n} \epsilon_{j s t} \frac{\partial^{2} T_{n t}}{\partial x_{m} \partial x_{s}} .
$$

Since the order of differentiation can be interchanged, it follows that if $\boldsymbol{S}$ is a symmetric tensor, then $\nabla \times(\nabla \times S)$ is symmetric.

The compatibility conditions in linearized elasticity are given by

$$
\nabla \times(\nabla \times \epsilon)=\mathbf{0} .
$$

Since $\epsilon$ is symmetric, the above equation has only six independent relations. In Cartesian coordinates, these relations are

$$
\begin{aligned}
& \frac{\partial^{2} \epsilon_{x x}}{\partial y^{2}}+\frac{\partial^{2} \epsilon_{y y}}{\partial x^{2}}-2 \frac{\partial^{2} \epsilon_{x y}}{\partial x \partial y}=0, \\
& \frac{\partial^{2} \epsilon_{y y}}{\partial z^{2}}+\frac{\partial^{2} \epsilon_{z z}}{\partial y^{2}}-2 \frac{\partial^{2} \epsilon_{y z}}{\partial y \partial z}=0, \\
& \frac{\partial^{2} \epsilon_{z z}}{\partial x^{2}}+\frac{\partial^{2} \epsilon_{x x}}{\partial z^{2}}-2 \frac{\partial^{2} \epsilon_{z x}}{\partial z \partial x}=0, \\
- & \frac{\partial^{2} \epsilon_{x x}}{\partial y \partial z}+\frac{\partial}{\partial x}\left(-\frac{\partial \epsilon_{y z}}{\partial x}+\frac{\partial \epsilon_{z x}}{\partial y}+\frac{\partial \epsilon_{x y}}{\partial z}\right)=0, \\
- & \frac{\partial^{2} \epsilon_{y y}}{\partial z \partial x}+\frac{\partial}{\partial y}\left(-\frac{\partial \epsilon_{z x}}{\partial y}+\frac{\partial \epsilon_{x y}}{\partial z}+\frac{\partial \epsilon_{y z}}{\partial x}\right)=0, \\
- & \frac{\partial^{2} \epsilon_{z z}}{\partial x \partial y}+\frac{\partial}{\partial z}\left(-\frac{\partial \epsilon_{x y}}{\partial z}+\frac{\partial \epsilon_{y z}}{\partial x}+\frac{\partial \epsilon_{z x}}{\partial y}\right)=0 .
\end{aligned}
$$


Southwell (1938) showed that all the six compatibility equations given by Eq. (11) are independent; it is not sufficient to verify (only) three of these equations in order to conclude whether a strain field is compatible. This finding is, of course, in direct contradiction to the claim by Borodachev (1995) that among the six conditions given by Eq. (12), only the first three or the last three conditions need be verified. We now demonstrate by a method different (and much simpler) than that of Southwell (1938), that the six compatibility conditions are indeed independent. Consider the strain field (i) $\epsilon_{x y}=x y$ with the remaining strain components zero. As can be seen from Eq. (12), only the first compatibility condition is violated with the remaining ones satisfied. Thus, for this strain field, only the first compatibility condition yields the conclusion that the given strain field is in fact incompatible. If the last three compatibility conditions are dependent on the first three (or vice versa), both sets should yield the same information about compatibility or incompatibility of a given strain field which is clearly not the case. We now simply extend this idea to show that each of the remaining compatibility equations are also independent. Thus, successively consider the fields where the only non-zero strain component is given by (ii) $\epsilon_{y z}=y z$, (iii) $\epsilon_{x z}=x z$, (iv) $\epsilon_{x x}=y z$, (v) $\epsilon_{y y}=x z$, (vi) $\epsilon_{z z}=x y$. It is easily verified that corresponding to each case, there is one compatibility equation that is violated while the remaining are satisfied. This shows that each equation in Eq. (12) yields information which the remaining five do not yield, thus proving that all six conditions are indeed independent.

Although it is not permissible to verify only three of the six compatibility relations given by Eq. (11), it is possible to formulate equivalent sets of three nonlinear compatibility relations as we now show. Let $S \equiv \nabla \times(\nabla \times \epsilon)$ (note that we have proved above that $S$ is symmetric), and let $\left(\lambda_{1}, \lambda_{2}, \lambda_{3}\right)$ and $\mathcal{I}_{S}$ denote the eigenvalues and principal invariants of $\boldsymbol{S}$. The six linear independent compatibility conditions can be stated as three nonlinear conditions

$$
\lambda_{i}=0, \quad i=1,2,3, \text { or } \mathcal{I}_{S}=\mathbf{0}, \quad \text { or } \operatorname{tr}(S)=\operatorname{tr}\left(S^{2}\right)=\operatorname{tr}\left(S^{3}\right)=0,
$$

since as shown at the beginning of this section,

$$
\boldsymbol{S}=\mathbf{0} \Longleftrightarrow \lambda_{i}=0, \quad i=1,2,3 \Longleftrightarrow \mathcal{I}_{S}=\mathbf{0} \Longleftrightarrow \operatorname{tr}(S)=\operatorname{tr}\left(S^{2}\right)=\operatorname{tr}\left(S^{3}\right)=0 .
$$

Although we have shown that, theoretically, it is possible to reduce the number of compatibility relations to three, in practice, it is easier to directly verify the six linear compatibility relations given by Eq. (11), since the explicit form of any of these three relations is quite cumbersome.

\section{Conclusions}

There is a growing literature that claims singularities, cavitation, interpenetration and other highly anomalous behaviour within the context of linearized elasticity even in the absence of cracks. It is shown in this work that such phenomena have nothing to do with the failure of linearized elasticity to satisfy material frame-indifference, and are merely the result of using faulty constitutive equations. By means of appropriate special loading cases, we deduce appropriate constraints that should be satisfied by the material constants, and which when obeyed, do not result in anomalous behaviour as seen from the work of Jain et al (1999). These constraints also resolve the long-standing issue that the constraint $1-v_{r}(r) v_{\theta}(r)>0$ imposes no constraints on the individual Poisson ratios $v_{r}$ and $v_{\theta}$, while in the isotropic case, it is known that $-1<v<0.5$.

It is shown that the linearized elastodynamics formulation for 'small on rigid' can be derived in a very simple manner by simply considering a frame of reference attached to the rigid motion. 
Further, it is shown that not neglecting $\boldsymbol{u}$ in comparison to $\boldsymbol{X}$, although not incorrect, yields no benefit in lieu of the added complexity that the resulting equations entail.

Finally, a much simpler demonstration has been provided to show that all the six compatibility relations are independent and that it does not suffice to verify only three of them. It has also been shown that it is possible to formulate the six linear compatibility relations in terms of equivalent sets of three nonlinear compatibility relations.

\section{References}

Borodachev N M 1995 Three-dimensional elasticity-theory problem in terms of the stress. Int. Appl. Mech. 31(12): 991-996

Ciarlet P G 1988 Mathematical Elasticity. North-Holland, Amsterdam

Fosdick R and Royer-Carfagni G 2001 The constraint of local injectivity in linear elasticity theory. Proc. Royal Soc. London, Series A 457: 2167-2187

Fosdick R, Freddi F, Royer-Carfagni G 2008 Bifurcation instability in linear elasticity with the constraint of local injectivity. J. Elasticity 90: 99-126

Freddi F and Royer-Carfagni G 2009 From non-linear elasticity to linearized theory: Examples defying intuition. J. Elasticity 96: 1-26

Galmudi D and Dvorkin J 1995 Stresses in anisotropic cylinders. Mech. Res. Commun. 22: 109-113

Horgan C O and Baxter S C 1996 Effects of curvilinear anisotropy on radially symmetric stresses in anisotropic linearly elastic solids. J. Elasticity 42 : 31-48

Jain R, Ramachandra K and Simha K R Y 1999 Rotating anisotropic disc of uniform strength. Int. J. Mech. Sci. 41: 639-648

Jain R, Ramachandra K and Simha K R Y 2000 Singularity in rotating orthotropic discs and shells. Int. J. Solids \& Structures 37: 2035-2058

Jog C S 2007 Foundations and applications of mechanics: Vol. I: Continuum mechanics. Alpha Science

Lekhnitskii S G 1968 Anisotropic Plates. Gordon \& Breach, New York

Marsden J E and Hughes T J R 1983 Mathematical foundations of elasticity. Prentice-Hall Inc., New York

Podio-Guidugli P 1987 The Piola-Kirchhoff stress may depend linearly on the deformation gradient. J. Elasticity 17(2): 183-187

Southwell R V 1938 Castigliano's principle of minimum strain-energy and the conditions of compatibility for strain. S. Timoshenko, 60'th Anniversary Volume, 211-217

Tang S 1969 Elastic stresses in rotating anisotropic disks. Int. J. Mech. Sci. 11: 509-517

Tarn J-Q 2002 Stress singularity in an elastic cylinder of cylindrically anisotropic materials. J. Elasticity 69: $1-13$

Ting T C T 1999a The remarkable nature of radially symmetric deformation of spherically uniform linear anisotropic elastic solids. J. Elasticity 53: 47-64

Ting T C T 1999b New solutions to pressuring, shearing, torsion and extension of a cylindrically anisotropic elastic circular tube or bar. Proc. Royal Soc. London, Series A 455: 3527-3542

Timoshenko S P and Goodier J N 1970 Theory of Elasticity. McGraw-Hill Book Company, New York

Tortorelli D A 2011 A generalized formulation of elastodynamics: Small on rigid. J. Elasticity 105(1-2): 349-363 